\title{
Prognosis of Heart Failure with Preserved Ejection Fraction in Primary Care by the H2FPEF Score
}

Antonio Jose Lagoeiro Jorge, ${ }^{\circledR}$ Maria Luiza Garcia Rosa, Wolney de Andrade Martins, ${ }^{\circledR}$ Adson Leite, Dayse Mary da Silva Correia, Maria Auxiliadora Nogueira Saad, Humberto Villacorta, ${ }^{\bullet}$ Sergio Chermont, ${ }^{\bullet}$ Ronaldo Altenburg Gismondi, ${ }^{\circledR}$ Breno Macedo Almeida, Evandro Tinoco Mesquita

Universidade Federal Fluminense, Niterói, RJ - Brazil.

\section{Abstract}

Background: Primary care physicians have difficulty dealing with patients who have HF with preserved LVEF(HFpEF). The prognosis of HFpEF is poor, and difficult to predict on primary care.

Objective: The aim of the study is to apply the H2FPEF score to primary care patients and verify its power to assess the risk of death or hospitalization due to cardiovascular disease.

Methods: This longitudinal study included 402 individuals, with signs or symptoms of HF, aged $\geq 45$ years and, underwent an evaluation which included clinical examination, BNP and echocardiogram. The diagnosis of HFpEF was confirmed by the criteria of the European Society of Cardiology. After five years, the patients were reassessed as to the occurrence of the composite outcome, death from any cause or hospitalization for cardiovascular disease. H2FPEF used six variables: body mass index, medications for hypertension, age, pulmonary artery systolic pressure, atrial fibrillation and E/e' ratio ranged from 0 to 9 points. The level of statistical significance was $\mathrm{p}<0.05$.

Results: HFpEF was diagnosed in 58(14.4\%). Among patients with $\mathrm{H} 2 \mathrm{FPEF} \geq 4,30 \%$ had HFpEF and in those with a score $\leq 4, \mathrm{HFpEF}$ was present in $12 \%$. Patients with HFpEF and H2FPEF $\geq 4$ had $53 \%$ of outcomes, whereas patients with HFpEF and a score $\leq 4$ had a $21 \%$ of outcomes. BNP values were higher in patients with HFpEF compared to those without $\mathrm{HFpEF}(\mathrm{p}<0.0001)$.

Conclusion: $\mathrm{H} 2 \mathrm{FPEF} \geq 4$ indicated a worse prognosis in patients with $\mathrm{HFpEF}$ assisted in primary care. H2FPEF may be a simple and useful tool for risk stratification in patients with HFpEF at the primary care. (Int J Cardiovasc Sci. 2020; 33(6):666-672)

Keywords: Heart Failure; Stroke Volume; Risk Assessment; Morbidity; Mortality; H2FPEF Score.

\section{Introduction}

After 18 years of the classic pathophysiological characterization of what we currently call "heart failure (HF) with preserved ejection fraction (HFpEF)", its diagnostic criteria remain questionable and evolving. ${ }^{1}$ Even with historically different diagnostic criteria, establishing its prevalence and assessing its prognosis is an increasing challenge in primary care. In 2016, the European Society of Cardiology (ESC) established the following as criteria for HFpEF: the presence of signs and or symptoms of HF; left ventricle ejection fraction (LVEF) greater than or equal to $50 \%$; elevation of natriuretic peptides; and the presence of cardiac structural or functional alteration. ${ }^{2}$ In general, primary care physicians find it difficult to deal with patients with multiple comorbidities, signs and symptoms of HF and preserved LVEF and, in such cases, easily accessible tools that assist the physician in diagnosis and prognosis can be extremely useful. Recently, a scoring system called the $\mathrm{H}_{2} \mathrm{FPEF}$ score ${ }^{3}$ was proposed to estimate the diagnostic probability of HFpEF in patients assisted in a specialized HF unit. However, the prognostic utility of this score remains 
unknown in primary care patients. The aim of the present study is to apply the $\mathrm{H}_{2}$ FPEF score to patients in primary care and to verify its predictive power of outcomes.

\section{Methods}

\section{Study Design}

We carried out a longitudinal study, derived from the DIGITALIS study, whose design has been previously published ${ }^{4}$, which included 402 individuals consecutively, aged 45 years or over, enrolled in primary care, in a city of 400,000 inhabitants, in Rio de Janeiro State, Brazil. Initial data were collected from July 2011 to December 2012 and the revaluation took place between January and December 2017, that is, five years later.

\section{Study Population}

The selection of the primary care units and the population was performed through random sequence generated by a computer program, where the power of each unit was proportional to the number of individuals assisted. We sent letters to the health staff to invite 1,050 individuals to participate in this study, and 666 of these individuals attended the visit and signed the consent form. Of those, 264 individuals were excluded, 33 individuals who did not complete all of the research procedures, 37 because they had LVEF less than 50\% and 194 because they did not present signs or symptoms of HF. The final study population was made up of 402 individuals (Figure 1).

Inclusion criteria were: age $\geq 45$ years, LVEF measured by the Simpson technique $\geq 50 \%$, signs or symptoms of HF and signing of the informed consent form. Individuals with disabilities to perform the procedures required for evaluation were excluded.

All subjects underwent anamnesis and clinical examination; laboratory tests, including B-type natriuretic peptide (BNP) dosing and tissue Doppler echocardiogram (TDE), all performed in a single visit.

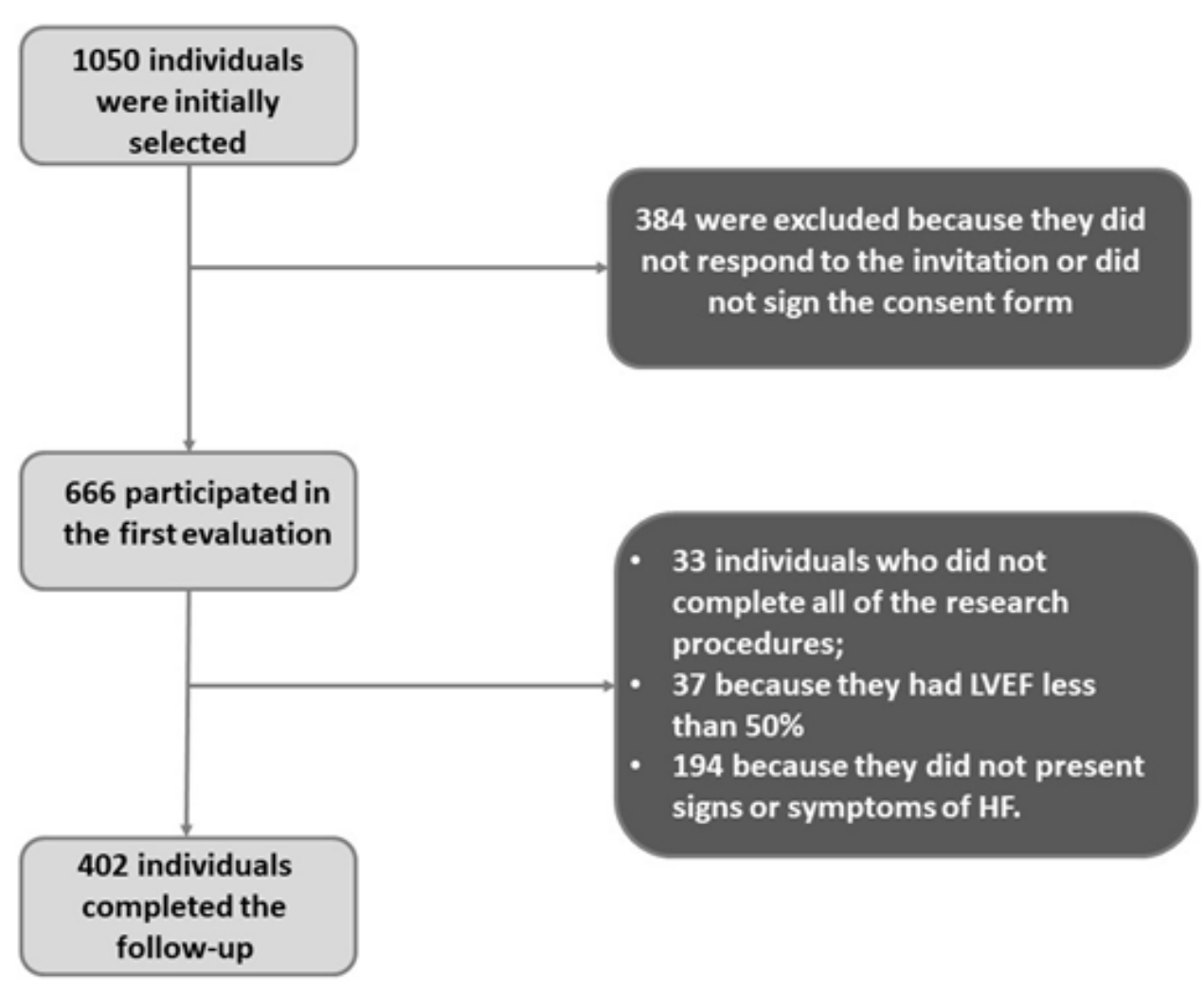

Figure 1 - Population selection flowchart. 
The TDE exams were performed by two echocardiographists without previous knowledge of the results of the other tests using two devices: Cypress 20 (Acuson, Siemens, USA) and AU-3 Partner (Esaote, Italy). The examinations were performed according to the recommendations for quantification of chambers of the American Society of Echocardiography (ASE) and the European Association of Echocardiography (EAE) .

\section{Outcome Measures}

The diagnosis of HFpEF was confirmed in individuals with signs and symptoms of HF, LVEF $\geq 50 \%$, elevation of BNP, and presence of structural alteration or diastolic dysfunction ${ }^{2}$. This evaluation was performed by two independent cardiologists who were blind to the study.

The $\mathrm{H}_{2}$ FPEF scoreuses six clinical and echocardiographic variables obtained in the evaluation of patients with $\mathrm{HF}$ symptoms. The variables were scored according to the strength of their respective association. The overall score of $\mathrm{H}_{2}$ FPEF ranged from 0 to 9 . The variables used and their score were: body mass index $>30 \mathrm{~kg} / \mathrm{m}^{2}$ ( 2 points); use of two or more drugs to treat hypertension (1 point); atrial fibrillation ( 3 points); PASP $>35 \mathrm{mmHg}$ ( 1 point); age $>60$ years ( 1 point); and high left ventricular filling pressures, E/e'> 9 (1 point) ${ }^{3}$ (Figure 2$)$.

After five years, the patients in this study were reassessed as to the occurrence of the composite outcome: death from any cause or hospitalization for cardiovascular disease, including decompensated HF, coronary artery disease, stroke and vascular diseases.

\section{Statistical Analysis}

Statistical analysis was performed with SPSS v 21.0 software (Chicago, Illinois, USA). Continuous variables were expressed as median and interquartile range, as none of them was positive for normality when tested using the Kolmogorov-Smirnov test. Categorical variables were expressed in absolute numbers and/or percentages. For comparison between groups (categorical variables), we used chi-square tests with continuity correction and Fisher's exact test when necessary. The Mann Whitney test was used to verify the existence

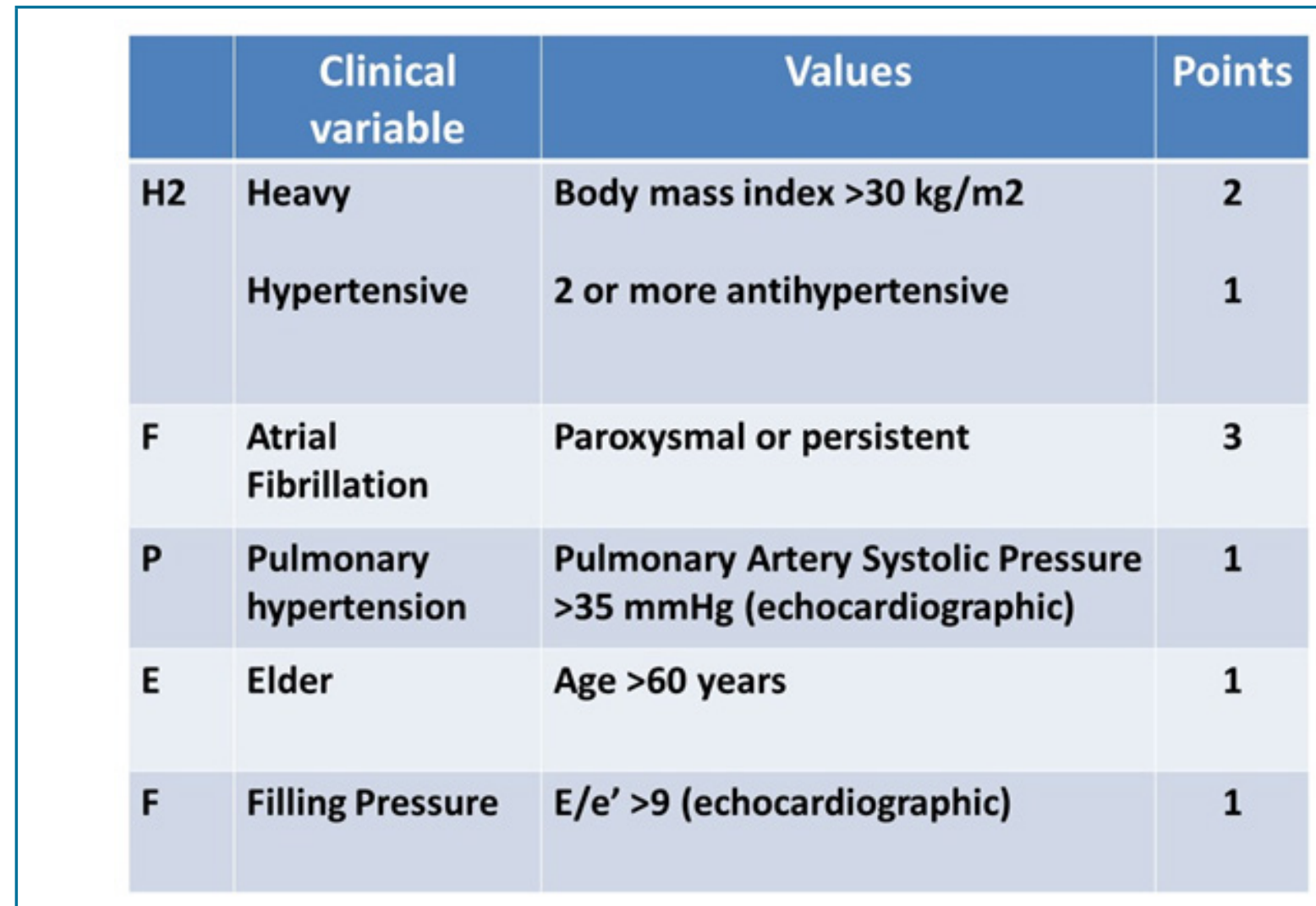

Figure $2-\mathrm{H}_{2}$ FPEF - score for each characteristic (maximum total of 9 points) 
of differences between continuous variables. In all comparisons, bilateral tests were performed and $\mathrm{p}$-values $<0.05$ were considered statistically significant.

\section{Ethical Considerations}

This study was conducted in accordance with the principles set out in the Declaration of Helsinki, revised in 2000 (Scotland 2000). The study protocol was approved by the Institution's Research Ethics Committee under the number 0077.0.258.000-10.

\section{Results}

Among the 402 subjects (mean age $=60.2 \pm 10.0$ years, $71 \%$ women) involved in the study, HFpEF was diagnosed in 58 subjects $(14.4 \%)$ and these patients with HFpEF had a score $\mathrm{H}_{2}$ FPEF 2 (1-4). All the parameters used in the score model were significantly different between patients with and without HFpEF, except for body mass index (BMI) and pulmonary artery systolic pressure (PASP). The anthropometric, clinical and laboratory characteristics are shown in Table 1. Table 2 shows the main echocardiographic parameters.

Among patients with a $\mathrm{H}_{2}$ FPEF score $\geq 4,30 \%$ had HFpEF and among patients with a score $\leq 4,12 \%$ presented HFpEF. BNP values were higher in patients with HFpEF compared to those without HFpEF, regardless of the score obtained in the $\mathrm{H}_{2} \mathrm{FPEF}$. In patients with HFpEF and score $\leq 3$, a lower outcome rate $(21 \%)$ was observed compared to patients with HFpEF and a score $\geq 4$ (53\%), showing that the higher the $\mathrm{H}_{2}$ FPEF score, the greater the risk of death and/or hospitalization due to cardiovascular disease.

After a 5-year follow-up, 42 (10.4\%) composite outcomes were observed, with $21 \%$ in patients with HFpEF and a score $\leq 4$, and $53 \%$ in patients with HFpEF and a score $\geq 4$. In patients without $\mathrm{HFpEF}$, the rate of outcomes was $7 \%$ in patients with a score $\leq 4$ and $6 \%$ in patients with a score $\geq 4$ (Table 3 ).

\section{Discussion}

This study evaluates the $\mathrm{H}_{2} \mathrm{FPEF}$ score in patients in primary care. Our data show that patients with HFpEF and $\mathrm{H}_{2} \mathrm{FPEF}$ score $\geq 4$ are at increased risk of death from any cause or hospitalization for cardiovascular disease.
A secondary analysis of the TOPCAT study evaluated the association between the probability of HFpEF by the $\mathrm{H}_{2} \mathrm{FPEF}$ score system and the primary endpoint composed of cardiovascular death, aborted cardiac arrest or HF hospitalization in patients with HFpEF using spironolactone or placebo. The high probability of HFpEF according to the $\mathrm{H}_{2}$ FPEF score was associated with worsening renal function, elevated natriuretic peptide values, increased left ventricle mass and left atrium (LA) size ${ }^{6}$.

When comparing the patients with and without HFpEF according to the $\mathrm{H}_{2}$ FPEF score, we observed that in those with a score $\leq 4$ there were significant differences between worsening renal function, increased left ventricle (LV) mass and LA volume index (LAV-I). However, in individuals with a score $\geq 4$, the difference was only observed in relation to LAV-I.

Although natriuretic peptides are part of the diagnosis of HFpEF in the ESC guideline, their values did not contribute the score. NT-proBNP values were missed in $24 \%$ of the patients enrolled in the elaboration of the score because some cardiologists did not request the test during clinical evaluation ${ }^{3}$. Our data show that BNP was higher in patients with HFpEF compared to individuals without $\mathrm{HFpEF}$, regardless of the $\mathrm{H}_{2} \mathrm{FPEF}$ score.

The Meta-Analysis Global Group in Chronic Heart Failure (MAGGIC) ${ }^{7}$, a risk score composed of 13 clinical variables, evaluated patients with HF across the spectrum of LVEF. Among the 13 variables used in the MAGGIC score, three were common to the $\mathrm{H}_{2}$ FPEF score (age, BMI and hypertension). The MAGGIC score did not use echocardiographic parameters and, like $\mathrm{H}_{2} \mathrm{FPEF}$, natriuretic peptides values were not considered. Of the patients defined with HFpEF ( $n=407)$, followed during $3.6 \pm 1.8$ years, $28 \%$ died, $32 \%$ were hospitalized for HF and $55 \%$ had cardiovascular hospitalization and/or death. When compared to our assessment of the $\mathrm{H}_{2} \mathrm{FPEF}$ score, we observed similar values in relation to the composite outcome observed in the MAGGIC score (55\%) and in $\mathrm{H}_{2} \mathrm{FPEF}$, with a score $\geq 4(53 \%)$. The $\mathrm{H}_{2} \mathrm{FPEF}$ score, like the MAGGIC, is a simple score, but with a smaller number of variables, and shows to be useful for morbidity and mortality risk stratification in HFpEF.

The study has limitations related to a single center design, the relative small sample size, with female predominance, and the score was applied retrospectively. Multicenter studies with large populations are needed to confirm our data. 
Table 1 - Anthropometric, clinical and laboratory characteristics of patients with and without heart failure.

\begin{tabular}{|c|c|c|c|}
\hline Variable & $\begin{array}{l}\text { Heart Failure } \\
\qquad n=58\end{array}$ & $\begin{array}{l}\text { No Heart Failure } \\
\qquad n=344\end{array}$ & p-value \\
\hline Age (years) & $71(60.6-77.2)$ & $57(51-64.7)$ & $<0.0001$ \\
\hline Women $(\%)$ & 78 & 70 & 0,346 \\
\hline BMI $\left(\mathrm{kg} / \mathrm{m}^{2}\right)$ & $27.7(23.3-30.6)$ & $28.1(25.2-31.8)$ & 0.111 \\
\hline SBP (mmHg) & 143.3(129.3-167.2) & 133(120-149) & 0.001 \\
\hline DBP (mmHg) & $80(71.7-91)$ & $82(73.7-90)$ & 0.689 \\
\hline HR (bpm) & $70(61-80)$ & $71.5(64.5-80)$ & 0.349 \\
\hline \multicolumn{4}{|l|}{ Risk factors } \\
\hline Smoking (\%) & 9 & 20 & 0.120 \\
\hline Diabetes (\%) & 29 & 29 & 0.523 \\
\hline Hypertension (\%) & 88 & 72 & 0.007 \\
\hline Obesity (\%) & 33 & 37 & 0.340 \\
\hline Metabolic Syndrome (\%) & 53 & 61 & 0.219 \\
\hline CKD (\%) & 53 & 15 & $<0.0001$ \\
\hline CAD (\%) & 15 & 9 & 0.102 \\
\hline Anemia (\%) & 22 & 10 & 0.014 \\
\hline \multicolumn{4}{|l|}{ Laboratory } \\
\hline Blood glucose (mg/dL) & 99.5(94-119) & 102(93-115) & 0.718 \\
\hline Hemoglobin (g/dL) & 13.2(12.4-14.5) & $13.5(12.7-14.4)$ & 0.445 \\
\hline Creatinine (mg/dL) & $0.86(0.72-1.05)$ & $0.82(0.70-0.96)$ & 0.024 \\
\hline GFR (mL/min) & $71.9((57.7-89.1)$ & $83.5(71.2-96.1)$ & $<0.0001$ \\
\hline Total Cholesterol (mg/dL) & 208(190-231) & $215(188-244)$ & 0.371 \\
\hline Triglycerides (mg/dL) & $112(87-143)$ & $120(88-179)$ & 0.184 \\
\hline HDL-chol (mg/dL) & $55(48-69)$ & $51(44-62)$ & 0.042 \\
\hline Albuminuria (mg/mL) & 11.8(5.9-28.6) & $12(6.6-25)$ & 0.819 \\
\hline Uric acid (mg/dL) & $4.9(4-6.1)$ & $5.1(4.1-6.2)$ & 0.714 \\
\hline BNP (pg/mL) & $54.5(42-93)$ & $14(10-23)$ & $<0.0001$ \\
\hline ACEI (\%) & 35 & 33 & 0.441 \\
\hline $\operatorname{ARB}(\%)$ & 14 & 11 & 0.362 \\
\hline Beta-blockers (\%) & 29 & 15 & 0.007 \\
\hline Diuretics (\%) & 50 & 34 & 0.017 \\
\hline \multicolumn{4}{|l|}{$\mathrm{H}_{2}$ FPEF Score components } \\
\hline Age $>60$ years $n(\%)$ & 78 & 41 & $<0.0001$ \\
\hline Hypertensive $\mathbf{n}(\%)$ & 53 & 38 & 0.018 \\
\hline $\mathrm{BMI}>30 \mathrm{~kg} / \mathrm{m}^{2} \mathrm{n}(\%)$ & 31 & 37 & 0.252 \\
\hline Atrial fibrillation n(\%) & 5 & 0 & 0.003 \\
\hline $\mathrm{E} / \mathrm{e}^{\prime}$ ratio $>9 \mathrm{n}(\%)$ & 28 & 12 & 0.003 \\
\hline PASP >35mmHg n(\%) & 3.5 & 0.5 & 0.056 \\
\hline $\mathrm{H}_{2}$ FPEF Score & $2(1-4)$ & $2(1-3)$ & 0.001 \\
\hline \multicolumn{4}{|c|}{$\begin{array}{l}\text { BMI: body mass index; BNP: B-Type natriuretic peptide; CAD: coronary artery disease; CKD: chronic kidney disease; DBP: diastolic blood pressure; } \\
\text { GFR: glomerular filtration rate by the MDRD equation; HDL-chol: HDL cholesterol; HFpEF: heart failure with preserved ejection fraction; HR: heart } \\
\text { rate; Hypertensive: } 2 \text { or more antihypertensive medicines; ACEI - angiotensin-converting-enzyme inhibitor; ARB - angiotensin receptor blocker; PASP: } \\
\text { pulmonary artery systolic pressure; SBP: systolic blood pressure. Continuous variables as median and interquartile range ( } 25 \text { and } 75 \%) \text {, overall p value } \\
\text { for continuous variables performed with the Mann-Whitney-U-test; Categorical variables presented as percentage (\%), p value for categorical variables } \\
\text { performed with Pearson's chi-square test. }\end{array}$} \\
\hline
\end{tabular}


Table 2 - Echocardiographic characteristics of patients with and without symptoms of heart failure.

\begin{tabular}{|c|c|c|c|}
\hline Variable & $\begin{array}{l}\text { Heart Failure } \\
\qquad n=58\end{array}$ & $\begin{array}{l}\text { No Heart Failure } \\
\qquad n=344\end{array}$ & p-value \\
\hline LVEF - Simpson (\%) & $61(57.7-65.2)$ & $61(58-65 \backslash 0$ & 0.790 \\
\hline LVMI (g/m²) & $105.4(93-121)$ & $88(75.9-120.4)$ & $<0.0001$ \\
\hline LAV-I (mL/m²) & 24.7(19.9-33.5) & $20.9(17.6-24.5)$ & $<0.0001$ \\
\hline E wave $(\mathrm{cm} / \mathrm{s})$ & $65.5(51.5-78.7)$ & 63(53.4-76) & 0.901 \\
\hline A wave $(\mathrm{cm} / \mathrm{s})$ & 79.5(58-93.8) & $68(56-82)$ & 0.019 \\
\hline $\mathrm{E} / \mathrm{A}$ ratio & $0.76(0.56-1.14)$ & $0.95(0.71-1.20)$ & 0.003 \\
\hline $\mathrm{S}^{\prime}$ wave $(\mathrm{cm} / \mathrm{s})$ & $8(6-9)$ & $8.5(7-9.5)$ & 0.021 \\
\hline $\mathrm{e}^{\prime}$ wave $(\mathrm{cm} / \mathrm{s})$ & $7.5(9.2-6.5)$ & $10(8-12)$ & $<0.0001$ \\
\hline $\mathrm{E} / \mathrm{e}^{\prime}$ ratio & $7.4(9.2-6.1)$ & $6.6((7.8-5.5)$ & 0.001 \\
\hline \multicolumn{4}{|c|}{$\begin{array}{l}\text { A wave: Mitral A velocity; E/A ratio: Mitral E/A ratio; } e^{\prime} \text { : Velocity of mitral annulus; E/e' ratio: Mitral E/e' ratio; E wave: Mitral E velocity; LAV-I: Lef } \\
\text { atrial volume index; LVEF: Left ventricular ejection fraction; LVMI: Left ventricular mass index; } S^{\prime} \text { wave: Peak annular systolic velocity. Continuous } \\
\text { variables as median and interquartile range ( } 25 \text { and } 75 \% \text { ), overall p value for continuous variables performed with the Mann-Whitney-U-test. }\end{array}$} \\
\hline
\end{tabular}

Table 3 - Outcomes in patients with and without heart failure with preserved ejection fraction according to H2FPEF score.

\begin{tabular}{|c|c|c|c|c|c|c|}
\hline & \multicolumn{2}{|c|}{$\begin{array}{l}\text { Score H2FPEF 0-3 } \\
\qquad(n=352)\end{array}$} & \multicolumn{4}{|c|}{$\begin{array}{c}\text { Score } \mathrm{H} 2 \mathrm{FPEF} \geq 4 \\
(n=50)\end{array}$} \\
\hline & $\begin{array}{l}\text { No HFPEF } \\
(n=309)\end{array}$ & $\begin{array}{l}\text { HFPEF } \\
(n=43)\end{array}$ & P-value & $\begin{array}{l}\text { No HFPEF } \\
\quad(n=35)\end{array}$ & $\begin{array}{l}\text { HFPEF } \\
(n=15)\end{array}$ & p-value \\
\hline Age (years) & $56(51-63)$ & $71(57-78)$ & $<0.0001$ & $64(61-67)$ & $73(66-77)$ & 0.002 \\
\hline Women n(\%) & $215(70)$ & $31(72)$ & 0.082 & $27(76)$ & $14(93)$ & 0.424 \\
\hline GFR (mL/min) & $84.2(71.7-96.1)$ & $74(58.3-88.9)$ & 0.001 & $75(64.5-86.9)$ & $64.4(45.5-90.1)$ & 0.315 \\
\hline $\operatorname{LVIM}\left(\mathrm{g} / \mathrm{m}^{2}\right)$ & 87.7(75.4-102.3) & $109.9(95.8-121.2)$ & $<0.0001$ & $93.5(80.6-105.8)$ & $93.8(78.4-104.2)$ & 0.874 \\
\hline LAV-I (mL/m²) & $20.8(17.4-24.2)$ & 23.7(19.1-30.7) & 0.001 & $21.8(19-26.9)$ & $26(21.4-36.1)$ & 0.044 \\
\hline $\mathrm{BNP}(\mathrm{pg} / \mathrm{mL})$ & $14(10-23.5)$ & $54(42-92)$ & $<0.0001$ & $11(10-23)$ & $57(46-97)$ & $<0.0001$ \\
\hline $\begin{array}{l}\text { Composite outcome } \\
\mathrm{n}(\%)\left(^{*}\right)\end{array}$ & $23(7)$ & $9(21)$ & 0.009 & $2(6)$ & $8(53)$ & $<0.0001$ \\
\hline \multicolumn{7}{|c|}{$\begin{array}{l}\text { GFR: Glomerular filtration rate by the MDRD equation; LAV-I: Left atrium volume index; LVIM: Left ventricular index mass; }\left({ }^{*}\right) \text { Death from any cause } \\
\text { and cardiovascular hospitalization. } \\
\text { Continuous variables as median and interquartile range ( } 25 \text { and } 75 \%) \text {, overall } p \text { value for continuous variables performed with the Mann-Whitney-U- } \\
\text { test; categorical variables presented as percentage }(\%), p \text { value for categorical variables performed with Pearson's chi-square test. }\end{array}$} \\
\hline
\end{tabular}




\section{Conclusion}

A $\mathrm{H}_{2}$ FPEF score $\geq 4$ indicates a worse prognosis in patients with HFpEF assisted in primary care. Our data show that a low-cost, easy-to-apply score, such as the $\mathrm{H}_{2} \mathrm{FPEF}$, can help physicians in primary care in the risk stratification of HFpEF patients.

\section{Author Contributions}

Conception and design of the research: Jorge AJL, Mesquita ET. Acquisition of data: Leite AR; Almeida BM; Correia D; Jorge AJL. Analysis and interpretation of the data: Jorge AJL; Martins WA. Statistical analysis: Rosa MLG; Jorge AJL. Writing of the manuscript: Jorge AJL; Villacorta Junior H; Martins WA. Critical revision of the manuscript for intellectual content: Martins WA; Mesquita ET; Saad MA.

\section{References}

1. Reddy YN, Borlaug BA. Heart failure with preserved ejection fraction. Curr Probl Cardiol. 2016;41(4):145-88.

2. Ponikowski P, Voors AA, Anker SD, Bueno H, Cleland JG, Coats AJ, et al. 2016 ESC Guidelines for the diagnosis and treatment of acute and chronic heart failure: The Task Force for the diagnosis and treatment of acute and chronic heart failure of the European Society of Cardiology (ESC). Developed with the special contribution of the Heart Failure Association (HFA) of the ESC. Eur J Heart Fail. 2016;18(8):891-975.

3. Reddy YNV, Carter RE, Obokata M, Redfield MM, Borlaug BA. A simple, evidence-based approach to help guide diagnosis of heart failure with preserved ejection fraction. Circulation. 2018;138:861-70.

4. Jorge AJL, Rosa MLG, Fernandes LCM, Freire MDC, Rodrigues RC, Correia DMS, et al. Estudo da Prevalência de Insuficiência Cardíaca

\section{Potential Conflict of Interest}

No potential conflict of interest relevant to this article was reported.

\section{Sources of Funding}

There were no external funding sources for this study.

\section{Study Association}

This study is not associated with any thesis or dissertation work.

\section{Ethics Approval and Consent to Participate}

This study was approved by the Ethics Committee of the Faculdade de Medicina da Universidade Federal Fluminense under the protocol number 49637115.7.0000.5243. All the procedures in this study were in accordance with the 1975 Helsinki Declaration, updated in 2013. Informed consent was obtained from all participants included in the study.

de Indivíduos Cadastrados no Programa Médico de Família - Niterói. Estudo DIGITALIS: desenho e método. Rev Bras Cardiol. 2011;24(5):320-5

5. Lang RM, Bierig M, Devereux RB, Flachskampf FA, Foster E, Pellikka PA et al. Recommendations for chamber quantification. Eur J Echocardiogr. 2006;7:79-108.

6. Segar MW, Patel KV, Berry JD, Grodin JL, Pandey A. Generalizability and Implications of the H(2)FPEF Score in a cohort of patients with heart failure with preserved ejection fraction. Circulation. 2019;139(15):1851-3.

7. Rich JD, Burns J, Freed BH, Maurer MS, Burkhoff D, Shah SJ. MetaAnalysis Global Group in Chronic (MAGGIC) Heart Failure Risk Score: Validation of a Simple Tool for the Prediction of Morbidity and Mortality in Heart Failure With Preserved Ejection Fraction. J Am Heart Assoc. 2018;7(20):e009594. doi: 10.1161/JAHA.118.009594. 